\title{
Enquête
}

Archives de la revue Enquête

6 | 1991

La socialisation de la jeunesse

\section{Jeunesse : objet politique, objet biographique}

\section{Thierry Blöss et Isabelle Feroni}

\section{(2) OpenEdition}

\section{Journals}

Édition électronique

URL : http://journals.openedition.org/enquete/147

DOI : 10.4000/enquete.147

ISSN : 1953-809X

Éditeur :

Cercom, Éditions Parenthèses

Édition imprimée

Date de publication : 2 juin 1991

Référence électronique

Thierry Blöss et Isabelle Feroni, « Jeunesse : objet politique, objet biographique », Enquête [En ligne], 6 | 1991, mis en ligne le 27 juin 2013, consulté le 10 décembre 2020. URL : http://

journals.openedition.org/enquete/147 ; DOI : https://doi.org/10.4000/enquete.147

Ce document a été généré automatiquement le 10 décembre 2020. 


\title{
Jeunesse : objet politique, objet biographique
}

\author{
Thierry Blöss et Isabelle Feroni
}

\section{NOTE DE L'AUTEUR}

Ce texte a pour origine un rapport de synthèse financé par l'Union des foyers de jeunes travailleurs (UfJT). Cf. bibliographie en fin d'article.

1 Les modes de catégorisation sociale constituent des objets d'analyse sociologiquement pertinents dans la mesure où, d'une part, ils constituent des instruments de classement des individus et de leurs comportements, et, d'autre part, ils sont au fondement des modes de traitement politique de ces individus.

2 Discours de sens commun et discours scientifique catégorisent différemment l'objet jeunesse, quand ce dernier n'essaie pas, par effet de mode, d'emprunter les schèmes classificatoires des représentations indigènes.

3 La définition commune de la jeunesse en tant que population ou groupe socioculturel d'appartenance illustre une tendance à l'institutionnalisation croissante des âges de la vie, et contribue à masquer la diversité des rapports sociaux dans lesquels sont pris les jeunes. L'appartenance de la jeunesse à une communauté d'âge contribue à produire l'illusion d'une catégorie homogène, tant du point de vue des besoins spécifiques qu'elle est censée exprimer que des problèmes non moins spécifiques qu'elle est censée rencontrer ou causer.

4 La définition sociologique de la jeunesse a principalement consisté à étudier les transformations sociales dont cette « catégorie » est l'objet. Cette voie d'élucidation n'est pas univoque, mais ouvre sur différents points de vue d'analyse réunis cependant par une démarche commune qui consiste à substituer au concept de jeunesse des notions qui désignent des enjeux sociaux particuliers. La jeunesse est tour à tour désignée comme une 
"post-adolescence ", un " passage à l'âge adulte », une " phase de cycle de vie », un lieu privilégié de « relations entre générations ».

5 «Jeunesse objet politique », il s'agira dans ce texte de comprendre la force idéologique du critère d'âge dans la définition sociale quotidienne, mais aussi dans la représentation et le traitement institutionnels dont elle fait l'objet.

6 "Jeunesse objet biographique ", il s'agira de comprendre dans quelle mesure la jeunesse peut être sociologiquement appréhendée autrement que comme une catégorie destinée naturellement à entrer dans l'âge adulte, et pour qui l'étude des modalités de ce passage est un moyen de la distinguer de ses aînés. La jeunesse n'est pas simplement une étape transitoire à problèmes. Elle constitue également un champ temporel d'observation des transformations dans les rapports de socialisation, notamment de sexe, et qui concernent l'ensemble du cycle de vie, quand la jeunesse ne constitue pas un terrain d'expérimentation de ces changements sociétaux.

\section{La jeunesse : objet politique}

$7 \quad$ La jeunesse est une catégorie qui fait l'objet d'une multiplicité de représentations sociales et de définitions institutionnelles. Aux représentations de sens commun qui l'appréhendent à partir de ses qualités individuelles s'opposent les représentations politiques centrées sur ses caractéristiques sociales.

8 À l'intérieur de chaque système de représentations, des critères spécifiques de description contribuent à structurer la perception de la jeunesse. Les discours de sens commun définissent la jeunesse principalement à partir de son âge. Indicateur de mesure temporelle de la jeunesse le plus couramment utilisé, l'âge est plus généralement institué en principe explicatif des comportements juvéniles.

9 Les discours de "sens politique " génèrent un processus d'étiquetage catégoriel de la jeunesse au centre duquel l'âge est un critère actif. Les politiques sociales définissent en effet couramment leurs publics d'intervention en termes d'âge. Sans compter qu'elles « objectivent» les attributs de la jeunesse par la mise en place de traitements sociaux correspondants. Appréhendés comme des facteurs clés de l'insertion des jeunes dans la vie active, le niveau de certification scolaire, mais également l'appartenance sociale à un espace local de résidence constituent, de ce fait, des critères déterminants de catégorisation institutionnelle de la jeunesse.

Dépassant leur fonction commune de critères d'identification sociale, l'âge, le niveau de certification scolaire et l'identité socio-spatiale deviennent ainsi de véritables opérateurs idéologiques. Ils contribuent à la structuration de véritables statuts sociaux spécifiques à la jeunesse. Objet de descriptions sociales multiples, porteuse d'enjeux contradictoires, la jeunesse constitue un objet politique à part entière.

\section{1. À travers l'âge : les représentations sociales de la jeunesse}

11 À travers l'histoire, les représentations sociales de la jeunesse ont pris des formes variées et contradictoires : une forme critique, les jeunes étant définis avant tout par défaut, par excès, ou encore par opposition au monde des adultes ; une forme positive, qui exalte et glorifie leurs capacités d'innovation et d'enthousiasme. Les perceptions sociales de la jeunesse sont donc ambivalentes, mettant l'accent tantôt sur sa capacité d'innovation, 
tantôt sur le danger potentiel qu'elle représente pour la cohésion du corps social. Selon les périodes historiques, l'une ou l'autre de ces formes de représentations a dominé, selon que le corps social avait intérêt à insister sur son unité ou au contraire sur sa volonté de changement. Les représentations de sens commun de la jeunesse constituent donc des évaluations subjectives de son intégration dans la société.

12 L. Thévenot (1985) a repéré la mise en œuvre de ce principe de qualification sociale contradictoire dans plusieurs champs de pratiques juvéniles. Les jeunes sont désignés comme "grands", lorsqu'ils sont productifs, qualifiés, et qu'ils maîtrisent les nouvelles technologies. Ils sont " petits » lorsqu'ils sont chômeurs, improductifs, non qualifiés. Le principe de la grandeur domestique qui reproduit la hiérarchie des générations oppose de même les "grands » dotés de l'autorité et de l'expérience aux «petits » immatures et novices. Le jeune est ainsi identifié à partir de ses faiblesses, de son inexpérience ou encore à partir de l'irrespect ou de l'« inconvenance » qu'il témoigne parfois à l'égard de cet ordre symbolique.

13 La jeunesse est souvent définie à partir des qualités morales qui lui sont imputées. Elle est également appréhendée essentiellement à partir du critère «triomphant » de l'âge. L'âge au même titre que le sexe figure, en effet, parmi les critères catégoriels les plus fréquemment employés pour décrire et compter les individus. Le repérage des personnes à partir de leur âge biologique permet d'ailleurs de faire l'économie d'une définition des classes d'âge par des critères relatifs aux modes de vie. Les intitulés «16-18 » ans ou «18-25 » ans se substituent à la désignation de ces catégories par leur problème, comme si l'appartenance à une classe d'âge était, en elle-même, la source principale des difficultés d'insertion. Être jeune ou vieux c'est d'abord avoir un certain nombre d'années. L'âge est devenu ainsi le principal critère de mesure du temps social (Thévenot, 1979).

14 Le recours à l'âge ne se limite pourtant pas à une fonction de description et de catégorisation des étapes du vieillissement social. Utilisé dans le cadre des interactions sociales (Widmer, 1983), l'âge fonctionne comme une ressource d'identification, permettant l'inférence d'éléments biographiques, et plus globalement leur interprétation. À un âge déterminé est en effet associée une compétence relative à certains types d'activités. L'indication de l'âge biologique des individus permet en conséquence de s'assurer de la normalité de leurs comportements, autorisant ainsi l'exercice d'une forme de contrôle social.

L'âge est donc tout à la fois un critère de catégorisation, une ressource d'identification biographique et un moyen de contrôle social. La définition et le traitement d'une population à partir du critère d'âge, par des instances investies d'un pouvoir de légitimation, contribue d'ailleurs à conférer une existence de fait à la catégorie ainsi constituée. Fonctionnant comme un critère d'ouverture de droits, l'âge ou plutôt l'appartenance à une classe d'âge permet de bénéficier d'un traitement social spécifique qui s'achève lors du franchissement de cette classe. Les politiques d'insertion en direction de « la » jeunesse agissent ainsi de façon catégorielle, limitant leur rayon d'action à des catégories d'âge étroitement définies, en excluant irrémédiablement les «vieux jeunes » censés avoir vu leurs problèmes ou difficultés d'insertion sociale disparaitre sous l'effet du vieillissement.

16 Cette approche catégorielle, qui confond les âges et les problèmes, ou plus exactement qui attribue à des classes d'âge étroitement définies des difficultés singulières, conduit en 
outre à sectorialiser la représentation et les modes de traitement social des différents âges de la vie, en multipliant ces derniers. L'enfermement des plus de 65 ans dans le statut de troisième âge, dérivé de la retraite, en constitue un exemple (Guillemard, 1972). L'âge constitue bel et bien un opérateur de définition et de traitement idéologique.

L'adoption d'une variable continue pour la description du temps biographique laisse cependant entier le problème des transitions entre les «âges de la vie ». La fluctuation des limites d'âge des publics juvéniles concernés par les politiques d'aide à l'insertion, entre 1980 et 1990, en constitue une concrète illustration. Le passage entre jeunesse et adultéité, loin d'advenir dans les limites clairement définies d'une tranche d'âge, se réalise au contraire à des moments différents selon les champs de pratiques considérés. Cherchant à situer la position de la jeunesse sur l'axe du temps, le taxinomiste amateur se trouve confronté à une multiplicité de systèmes de classement qui renvoient à autant de logiques contradictoires de distinction. Recensant les seuils d'âge qui autorisent le passage de la minorité à la majorité légale, J. Commaille (1985) constate le pluralisme juridique qui règne en la matière, et l'impossibilité qui en découle d'établir une conception universelle de l'adultéité ${ }^{1}$. L'accès à l'autonomie que confère l'état d'adulte s'opère donc de façon désynchronisée en fonction des divers systèmes de droits et des différents calendriers de pratiques.

Le classement généralement pratiqué en termes de classes d'âge repose en fait sur une approche biologique des âges de la vie. En s'attachant principalement à la description des phénomènes de croissance, cette approche définit la jeunesse comme la séquence biographique comprise entre la puberté et le vieillissement.

Avec le développement de la psychologie, la jeunesse a été analysée à l'aune de la crise de cette croissance. Nombre d'études ont en effet mis l'accent sur la crise d'adolescence, en tant que crise identitaire qui naîtrait d'un décalage - ou hysteresis - entre, d'une part la maturité physique et psychologique, et d'autre part la maturité sociale.

20 Mais le primat qui a été ainsi conféré à la notion de crise de l'adolescence pour expliquer l'essentiel des « problèmes » de comportements juvéniles, comme la délinquance, le refus de l'autorité parentale, voire même la contestation politique, a occulté les facteurs sociaux extérieurs à ces comportements.

«La référence explicite ou implicite à une définition bio- ou psychologique de la jeunesse conduit à imputer à une "nature jeune" des pratiques socialement déterminées, à occulter les différences d'origine, de situations, de devenirs sociaux, derrière l'identité présumée des dispositions psychologiques "jeunes", à accréditer l'idée qu'il existe une "nature" identique dont participent tous les jeunes et que l'unité du substantif ("la jeunesse") recouvre l'unité d'une substance.» (Lagrée \& Lew-Fai, 1983.)

21 Citant l'image classique des degrés d'âge, représentation allégorique des âges de la vie dans les anciens almanachs, J.-C. Chamboredon (1984) insiste sur l'insuffisance d'une définition de la post-adolescence en termes d'étape nouvelle qui viendrait s'intercaler entre l'adolescence et la jeunesse.

«Pour parler de la "post-adolescence", il ne suffit pas de parler d'une étape nouvelle de l'existence; il faut caractériser ce stade par des attributs (positions et droits dans divers champs institutionnels) socialement distribués, ce qui permet d'échapper à une représentation d'essence d'une période de la vie. Qu'est-ce qu'un post-adolescent? Quels sont ses attributs? Comment se définit la conjonction d'attributs qui définit un âge?» 

ions d'inscription urbaines de la jeunesse, les politiques d'insertion se caractérisen par une vision très ponctuelle et spécifique de ses difficultés d'insertion. Elles contribuent à « sédimenter » ces représentations par l'invention de statuts d'attente particuliers entre l'école et l'emploi. Les problèmes d'emploi et l'apparition de statuts nouveaux ne sont cependant pas limités à la seule catégorie de jeunesse. L'apparition de statuts intermédiaires entre l'emploi et le chômage s'inscrit, semble-t-il, dans une transformation d'ensemble de la population active. 


\subsubsection{Les politiques de formation et d'insertion socioprofessionnelle de la jeunesse} objectif de fournir un emploi que d'entretenir chez les stagiaires le sentiment que leur statut continuait à être proche d'une véritable situation d'emploi. Le lien d'activité était ainsi maintenu. Les politiques d'insertion ont donc rempli un rôle occupationnel, en inventant des statuts d'attente légalisés. La jeunesse a été en effet perçue comme une catégorie qui pouvait attendre. Il faut souligner toutefois la différence de signification que revêt cette période pour les différentes catégories de jeunesse. Si pour un étudiant la période prête à accumulation de diplômes et améliore les futures conditions d'insertion sur le marché du travail, pour un stagiaire inscrit dans un dispositif de traitement social, la période désigne une socialisation prolongée et concourt à aménager le temps de transition.

\subsubsection{L'espace résidentiel des jeunes : problèmes et besoins}

Les politiques publiques en direction de la jeunesse, après s'être focalisées dans un premier temps sur la problématique exclusive de la transition professionnelle, ont pris la 
mesure à partir de 1983 de la dimension territoriale des phénomènes d'insertion. L'espace résidentiel des jeunes va en effet apparaître à partir de cette époque comme le catalyseur essentiel des processus de socialisation familiale, scolaire et professionnelle. C'est à l'échelle du local que vont se conjuguer dès lors l'ensemble des actions entreprises en direction de la jeunesse.

Les objectifs de ces mesures sont multiples. Il s'agit tout d'abord de favoriser l'autonomie des jeunes primo-résidents dans leur accès à un logement par la création de structures intermédiaires jouant le rôle de caution morale et matérielle. Les orientations qui se dégagent de ces mesures sont destinées à corriger les inégalités sociales dont les jeunes ménages sont l'objet, notamment face au logement. Il s'agit également d'accroître les possibilités d'accès au parc locatif social, notamment à travers sa réhabilitation matérielle et sociale ${ }^{3}$. Il s'agit enfin d'inscrire l'aide à l'insertion sociale des jeunes dans l'espace territorial, en abordant de façon transversale ses différentes dimensions : école, emploi, logement, vie sociale et cadre de vie.

Par l'intermédiaire de ce dispositif, l'État se substitue donc aux solidarités familiales défaillantes, et instaure avec les jeunes une relation solidaire et individuelle. Qu'il s'agisse d'actions d'insertion professionnelle, résidentielle ou de loisirs, l'État semble prendre en charge «sur le mode parental» les rapports entre la société établie et les nouvelles générations de catégories défavorisées.

La politique en faveur du logement est révélatrice des modes de représentation et de traitement de la catégorie jeunesse. L'action de l'État dans l'aide à l'accession au logement des jeunes a désigné explicitement un public social cible, en difficulté. Sous l'intitulé d'une politique globale de la jeunesse, l'État a œuvré en réalité en faveur de l'insertion résidentielle et sociale de la jeunesse populaire, occultant ainsi les profondes inégalités de situation que connaissent les différentes jeunesses face au problème du logement.

Tout comme les politiques de l'emploi, les mesures d'aide à l'autonomisation résidentielle des jeunes ont traité l'insertion par le logement comme une étape ponctuelle. L'ensemble des aides proposées s'inscrit en effet, dans une courte durée. L'esprit du dispositif repose sur la volonté d'aider les jeunes à traverser le plus rapidement possible un moment difficile, afin qu'ils poursuivent ensuite, de façon autonome, leur trajectoire résidentielle. Pourtant la précarisation accrue des statuts d'insertion professionnelle modèle le processus de décohabitation juvénile, en multipliant les essais non aboutis, les échecs, et les tentatives réitérées sur le marché du logement.

Tout se passe comme si le caractère structurellement et durablement précaire du processus d'insertion des jeunes n'avait pas été mesuré à sa juste réalité, limitant ainsi l'efficacité des politiques aux seules catégories sociales de jeunes inscrits dans de véritables trajectoires d'insertion professionnelle. Ce qui a eu pour conséquence d'opérer une forme de sélection sociale du public juvénile. Le découpage en catégories d'âge, audelà desquelles l'aide au logement n'est plus envisagée ${ }^{4}$ a pour effet d'exclure du champ de l'intervention des politiques publiques le nombre chaque jour plus important de jeunes qui prolongent leur résidence commune avec leur famille d'origine et qui arrivent de ce fait plus tardivement sur le marché du logement.

Les représentations politiques de la jeunesse appréhendent enfin celle-ci comme une catégorie de consommateurs aux besoins spécifiques. Les jeunes auraient besoin de logements plus petits que les adultes, susceptibles d'être agencés, aménagés avec plus de liberté qu'un logement habituel, en vertu de la capacité créative attribuée aux jeunes. Or 
les différentes enquêtes réalisées jusqu'à maintenant indiquent que les jeunes n'éprouvent pas vis-à-vis du logement de besoins spécifiques. Leurs exigences de confort sont similaires en tout point à celles des adultes, notamment en ce qui concerne le rejet de la petitesse des logements.

Les dispositifs institutionnels ont donc réduit l'accès au logement des jeunes à des modes de vie standardisés, en se fondant sur une problématique spécifique des classes d'âge et en appréhendant les jeunes comme des monades isolées. Ils sont donc révélateurs d'une logique de traitement catégoriel de la jeunesse. Malgré l'intention de résolution transversale des problèmes juvéniles, les politiques du local ont reconduit l'appréhension naturaliste de la jeunesse, faisant d'elle une catégorie d'essence dotée de problèmes et de besoins particuliers.

\section{Jeunesse : objet biographique}

\subsection{La jeunesse: un « nouvel âge de la vie »?}

40 La définition politique de la jeunesse fait d'elle un groupe d'âge défini par des limites incertaines ou tout au moins variables selon les instances institutionnelles qui ont en charge la gestion de ses problèmes.

41 La notion d'âge constitue un opérateur idéologique de catégorisation et de traitement de la jeunesse en ce sens qu'elle préside à la représentation sociale de ses caractéristiques et difficultés, ainsi qu'à la production des services correspondants.

42 Les essais de définition sociologique de la jeunesse prennent également appui sur cette notion d'âge. Le point de vue est ici différent : la jeunesse est moins appréhendée comme une classe ou norme d'âge dotée de propriétés spécifiques, que comme une phase ou étape du cycle de vie, un champ temporel où se produisent des événements importants dans la vie, tels que l'entrée dans la vie matrimoniale, professionnelle et résidentielle adulte. Le terme de jeunesse désigne globalement dans ce cas un processus de passage au statut d'adulte, c'est-à-dire une période de transition entre deux périodes différenciées de l'existence.

Outre son extension à l'ensemble des couches sociales, selon des modalités sociohistoriques différenciées, la jeunesse est généralement appréhendée par son étirement temporel, facteur déterminant de ses transformations sociales internes.

Nombre d'enquêtes sociologiques ont constaté ce phénomène, que ce soit à travers l'observation de la prolongation de la scolarisation, de l'entrée plus tardive dans la vie active provoquée par l'accroissement des difficultés d'insertion professionnelle, ou encore du report de la primo-installation dans un logement autonome.

La mesure de ces différements constitue une étape importante dans l'analyse de la transformation des modes de vie. Mais cette sociologie des âges ne saurait occulter tout prolongement de réflexion qui tendrait à mettre l'accent sur le lien social existant entre les différents âges de la vie, sur les rapports intergénérationnels qui sous-tendent les problèmes sociaux rencontrés notamment par la jeunesse ; un lien qui ne saurait, quant à lui, réduire la jeunesse à un simple entre-deux-âges, un entre-deux-invariants biographiques que seraient d'une part l'enfance/adolescence et d'autre part l'âge adulte. 


\subsection{1. École, emploi, résidence : la mise en scène de l'étirement temporel de la jeunesse}

\section{La prolongation de la scolarisation}

46 remarquables de la transformation des modes d'entrée dans la vie adulte. Ce phénomène entraîne à la fois une hausse régulière du niveau de certification à la sortie du système de formation, et une entrée plus tardive sur le marché du travail. Cet allongement de la durée de scolarisation est d'abord visible à travers l'accroissement des effectiff ${ }^{5}$. La structure par âge des sortants du système éducatif s'en est trouvée modifiée ${ }^{6}$. La progression massive de la scolarisation des filles constitue un facteur notable de la démographie scolaire. Les filles sont actuellement plus jeunes que les garçons quand elles obtiennent le baccalauréat, et plus nombreuses dans l'enseignement supérieur? ${ }^{7}$. Leur taux de scolarisation entre 16 et 21 ans est également supérieur à celui des garçons. Le développement des scolarités féminines n'efface cependant pas les inégalités d'orientation. Les filles continuent à se diriger massivement vers des filières littéraires, et sont relativement peu représentées dans les formations scientifiques et techniques.

L'allongement de la scolarité et l'élévation du niveau de formation initial ne peuvent cependant être interprétés de manière univoque comme un mouvement de démocratisation de l'enseignement (Prost, 1986). L'extension sociale de l'enseignement s'est accompagnée d'une sélection accrue. Si les enfants d'ouvriers sont plus présents que par le passé dans le système éducatif, ils accèdent moins souvent en classe de seconde que les autres et sont plus souvent relégués dans l'enseignement professionnel.

angement de la scolarité, loin de concerner toute la jeunesse, a accru le clivage entre les jeunes issus des filières professionnelles courtes et ceux qui accèdent à l'enseignement supérieur. Les progrès de la scolarisation n'ont en effet modifié que les extrémités de l'échelle des diplômes obtenus à la sortie de l'appareil d'enseignement ${ }^{8}$. L'augmentation des diplômés de l'enseignement supérieur provoque des réactions en chaîne sur l'échelle sociale de la certification, en contribuant à la dépréciation des diplômes de niveau inférieur. Le processus d'inflation des diplômes entraîne, en effet, la dévaluation de l'ensemble des titres scolaires (Passeron, 1982) et affecte de façon d'autant plus intense ceux qui en sont dépourvus. Les jeunes non diplômés se retrouvent ainsi les plus pénalisés lors de leur entrée sur le marché du travail ${ }^{9}$. C'est ainsi toute l'échelle de la rentabilité sociale des diplômes qui connaît une mutation: le niveau scolaire correspondant à deux ou trois années au-delà de la scolarité obligatoire, soit CAP, BEP ou $\mathrm{BAC}$, tend à devenir le niveau de référence minimal pour prétendre à un emploi qualifié.

La généralisation de la " post-adolescence » (Chamboredon, 1984), ou période transitoire importante entre l'école et l'emploi, pourrait laisser croire à l'extension du modèle bourgeois de la jeunesse, entre-deux-âges marqué par l'inactivité professionnelle. Cet allongement temporel recouvre en fait des trajectoires sociales très dissemblables. Alors que l'espérance de vie scolaire s'accroit pour l'ensemble des jeunes, les filières scolaires empruntées par ces derniers ont des valeurs sociales différentes, et leurs points d'arrivée placent les individus dans des positions inégales face aux possibilités d'accès au marché de l'emploi. 


\section{Dans l'attente d'un emploi : chômage et précarité}

d'un statut professionnel définitif ou « stable ». L'allongement de la scolarisation juvénile conduit les jeunes à rentrer à un âge plus tardif sur le marché du travail ${ }^{10}$. Le chômage mais aussi la précarisation des statuts d'emploi, la multiplication des statuts d'attente allongent la période d'insertion professionnelle et contribuent à différer dans le temps l'accession à l'autonomie individuelle. Un certain nombre d'analyses ont d'ailleurs proposé de substituer au concept d'insertion celui de transition professionnelle, qui serait mieux à même de décrire la complexité du passage entre l'école et l'emploi avec ses alternances de périodes de chômage, d'emplois précaires, de formation (Bouffartigue, Lagrée \& Rose, 1989). Ce bouleversement des modes de transition entre les âges, dont la forme la plus visible est la situation d'incertitude face à l'emploi, est d'autant plus ressenti par les plus démunis que son issue peut en être l'exclusion durable de toute forme d'insertion sociale, selon un cycle vicieux de la marginalisation sociale.

Depuis une quinzaine d'années, la crise a surtout touché les débutants sur le marché du travail. Bien qu'entrant plus tardivement en activité, et ce avec une qualification moyenne plus élevée que leurs aînés, les jeunes sont pénalisés par le chômage ${ }^{11}$. Jeunes qualifiés et non qualifiés se différencient également, ces derniers formant le noyau dur du chômage. Ce sont les jeunes dépourvus de diplômes qui ont connu au cours de ces dernières années l'augmentation des risques de chômage la plus sensible et l'expérience d'emplois déqualifiés à statut précaire.

Pour pallier l'effondrement, au cours de ces dernières années, de l'embauche de jeunes débutants dépourvus de qualification, directement au sortir de l'école, un traitement social de l'emploi juvénile a été mis en place. Son développement ${ }^{12}$ n'est pas étranger à la précarisation croissante de l'activité professionnelle des jeunes, que cette précarisation s'exprime à travers le développement des contrats à durée déterminée et de l'intérim ${ }^{13}$, comme nous l'avons précédemment mentionné, ou encore par le développement du sousemploi ${ }^{14}$, autre forme de précarité professionnelle particulièrement juvénile qui s'accompagne d'une diminution des emplois classiques à temps complet. Loin d'être un temps choisi, ce travail à temps partiel est un temps contraint, une solution acceptée faute de mieux pour sauvegarder un emploi. choisi, est en réalité une des expressions de la flexibilité de l'emploi qui s'exerce au détriment des catégories d'actifs les plus fragiles : les jeunes et les femmes.

" Aujourd'hui donc, dans de nombreux secteurs, lorsque l'on parle du temps partiel,

il ne s'agit ni de désirs ni d'aspirations, mais de gestion de la flexibilité. » (Maruani

\& Nicole, 1989.)

L'importance du sous-emploi juvénile n'est pourtant pas un fait nouveau. Ce qui a changé tient à la nature des secteurs d'activité générateurs de ce sous-emploi. Les stages et autres TUC en milieu associatif, au sein des collectivités territoriales et dans les administrations publiques alimentent aujourd'hui en majeure partie le flux de ce sous-emploi. Le sousemploi juvénile est devenu une affaire... d'État. 


\section{Quitter plus tardivement ses parents} serait un âge intermédiaire entre d'un côté, le monde de l'enfance/adolescence réglé et codifié par des relations de dépendances institutionnelles, incarnées principalement par 
les rapports d'éducation familiale et de scolarisation, et, d'un autre côté, le monde des adultes, qui placerait l'individu, et au-delà les personnes qui lui sont liées (conjoint) ou redevables (enfants), face à de lourdes responsabilités sociales. Gare aux effets sociaux du chômage adulte qui dépasseraient le cadre individuel des personnes concernées! À croire, si l'on se fie aux sens commun et scientifique (complices pour une fois !) qu'il vaut mieux qu'un jeune soit au chômage qu'un adulte, le tribut social n'étant apparemment pas le même pour la société.

La jeunesse serait, dans cette vision du monde largement répandue, une période... prolongée d'adaptation, de tâtonnements professionnels, d'incertitude amoureuse, etc.; une période où l'on quitte un mode d'existence fortement réglé par les aînés et où l'on découvre, par étapes successives, mais de plus en plus aléatoires, un monde de responsabilités. Et dans ce jeu d'ajustement biographique, on s'accorde volontiers à reconnaître à la jeunesse de grandes qualités ou capacités d'adaptation. Plus que n'importe quelle autre catégorie de population, la jeunesse est attendue s'ajuster, par sa souplesse d'esprit et de corps, aux réalités sociales du moment. Sans compter que l'efficacité de cet ajustement est notamment due au fait qu'il est intériorisé par les jeunes et par l'ensemble du corps social comme étant avant tout une affaire d'individus. Nombreux sont les paramètres qui indiquent ou précisent le caractère apparemment strictement personnel de ce processus d'ajustement: on s'ajustera d'autant mieux que l'on sera diplômé, volontaire, mobile, discipliné, etc.

63 Ce mode de perception de la jeunesse comme un âge de la vie, comme un processus de passage à l'âge adulte, n'est pas sans intérêt heuristique, notamment celui de reconnaître que la phase de jeunesse est assortie d'événements plus ou moins maîtrisés ou librement acceptés (sortie de l'école avec un niveau de succès ou d'échec à la clé ; départ de chez les parents plus ou moins volontaire, par anticipation ou non,...), qui vont placer l'individu sur des pentes sociales différenciées ${ }^{17}$.

64 Ce mode de perception souffre également d'un certain nombre de critiques dont la plus importante est, selon nous, d'appréhender de façon évolutionniste la catégorie de jeunesse au sein du cycle de vie, c'est-à-dire comme un âge moratoire marqué par des retards ou « différements » de comportements... d'ajustements (Galland, 1984).

Constater que l'évolution sociologique de la jeunesse correspond à l'évolution temporelle des calendriers scolaires, professionnels, résidentiels et familiaux, est probablement insuffisant. Ce constat doit être complété par le fait que cette évolution correspond tout autant à une transformation qualitative des pratiques d'insertion.

Chaque événement connaît des changements internes qui affectent l'ensemble des profils d'entrée dans la vie adulte : les différents événements qui se commanditaient les uns les autres jusque dans les années 1970 se déconnectent quelque peu ou du moins redéfinissent leurs relations mutuelles. La transition professionnelle plus aléatoire offre à lire l'invention de nouveaux statuts socioprofessionnels; l'autonomisation résidentielle plus tardive se fonde sur de nouveaux modes de décohabitation, plus solitaires. L'élévation de l'âge au mariage laisse apparaître de nouveaux modes d'unions conjugales.

Sans compter que ces nouvelles tendances sociologiques et démographiques, loin de coller à des modes d'exister juvéniles, constituent une grille de lecture des changements intervenus dans le «monde des adultes" lui-même. La précarité professionnelle expérimentée par la jeunesse ne s'est-elle pas étendue aux classes d'âges supérieures, initiant ainsi une transformation sensible des arbitrages et des rapports au sein du 
collectif de travail ? Les « nouveaux » statuts matrimoniaux expérimentés par la jeunesse (cohabitation juvénile, célibat prolongé), qui consacrent généralement une baisse de la vie en couple, ne sont-ils pas à mettre en relation avec les changements sociodémographiques qui mettent en question le rôle des rapports d'alliance dans la parentalité (développement des familles monoparentales après divorce,...)? Autant d'exemples qui illustrent l'idée que les transformations de la jeunesse préfigurent des changements structurels qui dépassent son propre cadre socio-temporel.

\subsection{La définition de la jeunesse : une question de rapports intergénérationnels}

Appréhender la jeunesse non plus comme un groupe social plus ou moins homogène ou culturellement autonome, mais comme un processus de passage à l'âge adulte permet sans doute de privilégier la dimension «processuelle» ou biographique de cet état. La socialisation de la jeunesse, sa définition en tant qu'âge de la vie ne met pourtant pas l'analyste à l'abri d'une vision par trop naturaliste selon laquelle la jeunesse ne serait que suite chrono-logique de l'adolescence, une l'étape préparatoire de l'adultéité.

Quand la jeunesse est généralement pensée comme constituante de la structuration biographique du cycle de vie, comme une cohorte ou génération, un point de vue semblet-il prévaut, ainsi résumé : la jeunesse se définirait comme un processus caractérisé par une série d'engagements de plus en plus nombreux envers les normes et les institutions conventionnelles. La socialisation de la jeunesse désigne principalement dans ce cas - conformément au paradigme durkheimien des rapports d'éducation intergénérationnelle, fondé sur le principe de séniorité - une relation unilatérale faite de la transmission des valeurs culturelles, c'est-à-dire de l'adhésion des plus jeunes aux valeurs prônées par la société des aînés, de leur intégration aux normes de l'ordre social.

70 L'usage du concept de génération est ici au centre de notre réflexion; un usage sociologique précis qui entend apporter un éclairage analytique nouveau à la notion de jeunesse. L'approche générationnelle présenterait en effet l'intérêt de situer les pratiques d'accession à l'âge adulte dans le temps biographique et historique, en évitant l'écueil essentialiste d'une représentation de la jeunesse en termes de classe d'âge. La prise en compte des rapports intergénérationnels présenterait également l'intérêt de mieux décrire les rapports sociaux qui structurent cette dite « étape de la vie».

71 Nous n'exposerons pas ici « le débat long et ardu qui reflète l'histoire mouvementée » de la notion de génération, pour nous en tenir à son application heuristique dans le champ de la jeuness $\mathrm{e}^{18}$. Nous envisagerons particulièrement comment le concept de génération peut aider à la compréhension des comportements juvéniles en les situant dans l'ensemble des rapports de socialisation familiaux et extra-familiaux.

\subsubsection{La forme générationnelle de la jeunesse : éléments d'analyse classificatoire}

72 L'utilisation de l'approche générationnelle pour l'analyse des comportements d'insertion pose un certain nombre de problèmes méthodologiques et conceptuels (Godard, 1985). Le premier d'entre eux porte sur la définition même du concept de génération. Selon les auteurs, on désigne sous ce terme des groupes sociaux concrets dotés de la conscience d'appartenir à une même génération ou des cohortes socialisées dans des conjonctures historiques semblables. 
«Utiliser la notion de génération, c'est-à-dire réunir sous le même nom tel ensemble d'hommes qui ont à peu près le même âge [...] c'est supposer, soit que cet ensemble d'hommes, qui sont à peu près du même âge, forme un "groupe mobilisé", partageant les mêmes représentations, dispositions et pratiques, soit qu'il constitue un "groupe mobilisable", uni au moins par le sentiment de contemporanéité, un état d'âme, une mentalité des souvenirs, des réminiscences. » (Mauger, 1985a.)

Dans la deuxième hypothèse, le terme de génération recouvre des cohortes historiques, c'est-à-dire un ensemble d'individus qui ont connu au même moment, c'est-à-dire aux mêmes âges ou plus précisément encore à des moments comparables du cycle de vie, les mêmes événements, qu'ils soient historiques, politiques ou économiques voire culturels. On peut ainsi parler des générations de la guerre, de « 68 » ou plus récemment de la crise. Le terme de génération désigne des cohortes qui ont en commun des expériences historiques ou collectives.

4 C'est ainsi que la génération de la crise se caractérise, par l'expérience commune d'un contexte particulier d'accession à l'âge adulte (prolongation de la scolarisation, difficulté accrue de l'insertion professionnelle, précarisation des formes d'emploi), et par la mise en place de modèles nouveaux d'autonomie sociale (décohabitation tardive, intensification du célibat, différement de l'installation matrimoniale).

5 L'analyse générationnelle permet de comparer les pratiques de plusieurs générations situées dans les mêmes classes d'âge et de rendre ainsi compte de la périodisation différente des cycles de vie en fonction des époques. Le « différement » dans le temps des calendriers de décohabitation, d'installation matrimoniale et de stabilisation professionnelle oppose de façon très nette les générations du «baby-boom » et de «la crise $»^{19}$. La mise en perspective générationnelle dans l'évolution des revenus montre que si les revenus des jeunes classes d'âge (rapportés au SMIG du moment) sont en règle générale inférieurs aux revenus des plus âgés, les générations entrées sur le marché du travail en 1982 ont la particularité d'avoir des revenus inférieurs à la génération entrée sur le marché du travail en 1977 (CERC, 1989). La conjoncture économique crée, pour des générations successives, des conditions différentes d'entrée sur le marché du travail.

Le concept de génération, au-delà d'une simple contextualisation historique ou conjoncturelle des pratiques, permettrait donc de rendre compte des évolutions structurelles qui affectent les différents moments du cycle de vie et plus particulièrement le passage à l'âge adulte.

La génération d'appartenance rend compte de l'expérience commune d'une conjoncture historique ou économique lors de l'accession à l'âge adulte, sans unifier pour autant les formes et le contenu de ces expériences qui demeurent socialement différenciées. À l'intérieur d'une génération, il y a en effet contraste ou différenciation sociale. Cette réalité a été sous-évaluée ou négligée par les premiers travaux de sociologues et de démographes dont la tâche a consisté à fonder le plus rationnellement possible l'appartenance à une génération, autrement dit à sélectionner les critères d'agrégation des individus à cet ensemble. C'est la raison pour laquelle de nombreux travaux se sont attachés à décider de métriques ou d'intervalles temporels ( 5 ans, 10 ans, etc.) ${ }^{20}$ afin de déterminer les principes d'unité générationnelle, considérant ainsi la notion de génération comme une variable continue.

78 Au même titre que la notion de jeunesse, le concept de génération porte donc en lui la dérive possible d'une lecture catégorielle. Nous considérerons qu'il ne peut constituer un principe d'explication univoque auquel pourraient être rapportés les comportements de 
ses membres. Une génération est un groupe traversé par des différences d'origine sociale, de sexe et de lieu,...

\subsubsection{Histoire biographique et identité juvénile}

79 La représentation sociale de la jeunesse, tant dans les discours institutionnels que dans le langage de sens commun, masque deux objets sociologiques essentiels que sont l'histoire biographique et l'appartenance socio-historique. Le sujet juvénile des pratiques doit être situé dans ce double contexte qui concourt à la définition de son identité individuelle et collective.

80 À examiner de près les actions sociales en direction de la jeunesse, un paradoxe peut être relevé qui consiste à considérer le sujet juvénile des pratiques en tant que tel, lorsque les besoins exprimés concernent des questions factuelles telles les loisirs, la mode,... et à l'appréhender à partir de facteurs extérieurs à son individualité, lorsque ces questions désignent des enjeux majeurs d'insertion sociale, telle l'entrée dans un emploi ou dans un logement.

81 Dans le premier cas, le sujet juvénile suffit à l'expression et à la reconnaissance identitaire de ses besoins. Dans le second cas, cette reconnaissance a pour médiation son appartenance sociale. Dans la pratique, le traitement social de la demande juvénile n'est pas étroitement limité aux jeunes, mais prend en compte les critères d'appartenance socio-familiale. Dans le champ du logement social par exemple, l'examen des candidatures juvéniles s'effectue au travers du prisme familialiste. L'appréciation de la bonne qualité de la candidature est subordonnée au passé locatif des parents du demandeur. C'est la réputation de la famille d'origine et non plus les besoins du jeune luimême qui devient le critère déterminant de la sélection. La candidature traitée est une candidature familiale, sociale et non une candidature juvénile.

L'appartenance et le positionnement générationnels constituent des dimensions à part entière de l'identité individuelle mais aussi collective de la jeunesse. L'étude de la sociabilité des jeunes urbains en milieu populaire nous a permis de le vérifier (Blöss, 1989). À partir des années 1980 , dans le cadre des opérations de développement social des quartiers, la prise de parole et l'engagement participatif des jeunes dans la gestion sociale des quartiers ont été suscités par les instances politiques. Dans ces quartiers suburbains, les bandes informelles de jeunes ont cédé partiellement la place à des groupes officiellement structurés en associations. Un des objectifs était de responsabiliser la jeunesse précarisée en prenant en compte les réseaux de sociabilité existants, notamment les réseaux de parenté élargis aux collatéraux.

83 Ces nouvelles formes de sociabilité résidentielle ont été interprétées, dans l'idéologie ambiante, comme l'expression d'une culture de jeunes sensibles aux nouvelles communications des sociétés post-urbaines. Or, l'analyse de la composition sociale et ethnique de ces associations des jeunes issus de l'immigration montre que ce qui est perçu comme de l'identité juvénile prend en fait sa source dans des trajectoires sociofamiliales. L'expression associative de ces jeunes témoigne ici de leur préoccupation de ne plus être laissés pour compte, comme leurs parents l'ont été pendant des décennies, et de voir à l'avenir leurs enfants et leurs petits frères et sœurs connaître un meilleur destin. L'histoire familiale et résidentielle des jeunes, leur conscience générationnelle d'appartenir à un moment de cette histoire constituent par conséquent un facteur au 
moins aussi décisif que l'action des politiques sociales pour expliquer l'émergence de leurs nouveaux comportements en matière de sociabilité résidentielle.

Le positionnement générationnel, la mémoire de l'itinéraire biographique accompli par la famille structurent dans ce cas les comportements individuels et collectifs. La notion de génération désigne ici, par conséquent, des groupes partageant un certain nombre de représentations sociales dont les fondements sont probablement à rechercher dans leur mémoire du passé, mémoire historique, mémoire biographique, dans tous les cas, «mémoire collective ».

Cette mémoire collective est un héritage. Dans les quartiers populaires, existe en effet un "héritage du pauvre", selon l'expression de G. Mauger (1989) qui est de l'ordre de la «mémoire ». De mémoire de famille... !

\subsubsection{La transformation des rapports de socialisation}

L'approche générationnelle n'est pas récente. Mais les travaux réalisés ${ }^{21}$ jusqu'alors ont privilégié la dimension morphologique et politique de la jeunesse. La jeunesse, en tant que mouvement social constituerait, selon cette optique, "une force de changement social, i. e. une sorte d'élite au sens de la sociologie politique » (Godard, 1991).

Analyser la jeunesse en tant que génération, c'est également s'interroger sur les liens entre ses membres; c'est aussi et surtout s'intéresser à la multiplicité et à l'intensité des rapports que ces individus tissent avec les autres catégories d'individus. Comme tout critère de classement ou de "division du monde social », la notion de génération désigne des groupes constitués par la nature des rapports sociaux qu'ils nouent entre eux. Les rapports de socialisation familiale constituent un cadre privilégié pour observer la polysémie de ces rapports intergénérationnels.

L'approche générationnelle permet concrètement de souligner les effets des rapports entre générations sur la construction et le traitement social de la jeunesse. Loin de s'opérer dans un vide social à l'abri de tout contact inter-âge, ou à l'intérieur d'un groupe de pairs mythiquement replié sur lui-même et séparé du monde, la construction de la jeunesse s'opère constamment dans un réseau actif de rapports sociaux qui met aux prises plusieurs générations, notamment celle des parents avec celle des enfants.

Nous évoquerons ici trois types de rapports sociaux intergénérationnels auxquels est confrontée la jeunesse : les rapports de solidarité, de conflit, et de concurrence ou rapports de succession économique, professionnelle entre générations pour l'occupation des places sociales.

90 La dynamique des rapports intergénérationnels se cristallise dans des systèmes de droits et de devoirs de certaines catégories d'âge par rapport à d'autres. J. Commaille (1985) remarque d'ailleurs que dans le droit, la jeunesse n'apparait jamais

« comme catégorie en soi, état en soi, mais comme une phase constamment mise en rapport avec des éléments en amont et en aval du processus de relations entre les générations, relations médiatisées par des institutions ».

91 Les rapports intergénérationnels qui structurent la jeunesse sont le plus souvent envisagés du point de vue de l'aide dont elle bénéficie de la part des plus âgés. Les rapports de solidarité familiale s'exprimeraient ainsi dans le sens de la filiation. Il s'agit des parents qui aident leurs enfants à trouver un emploi, qui fournissent une aide matérielle lors de la première installation résidentielle, ou qui accueillent leurs enfants à l'issue d'un essai matrimonial non réussi. 
92 Plusieurs formes de solidarités caractérisent d'ailleurs la problématique de l'insertion résidentielle. Le maintien au domicile parental des jeunes chômeurs de familles populaires peut être interprété comme une forme de solidarité matérielle entre générations, alors que la recohabitation des étudiants des catégories moyennes et supérieures s'inscrit dans des trajectoires de certification scolaire. L'aide reçue n'a pas la même signification sociale dans les deux situations évoquées. Dans le premier cas, il s'agit d'éviter la marginalisation économique et sociale des enfants, ce qui a pour conséquence de retarder leur installation résidentielle. Dans le second cas, il s'agit de poursuivre l'effort familial de promotion sociale en permettant aux enfants un jeu d'essais/erreurs « sur les marchés » de l'insertion (Blöss, Frickey \& Godard, 1990).

La montée de la crise économique a exacerbé l'importance des rapports de solidarité intergénérationnelle lors du passage à l'âge adulte. On apprécie le caractère décisif du soutien familial pour l'insertion sociale des enfants précisément quand on mesure les conséquences que sa défaillance peut entraîner. C'est ainsi que nombre des jeunes chômeurs que nous avons rencontrés ${ }^{22}$ appartiennent à des familles si socialement affaiblies qu'elles se trouvent dans l'impossibilité d'aider leurs enfants. Dans certains cas, ce sont les parents qui sollicitent un soutien de la part de leurs enfants, ou en bénéficient; ce qui peut avoir pour effet de rendre socialement captifs ces derniers: captivité résidentielle, le jeune restant plus longtemps à demeure familiale; captivité matérielle, les conditions de sa prise d'autonomie étant rendues plus difficiles. Les relations de solidarité peuvent ainsi inverser leur sens filiatoire habituel pour s'exercer au profit des ascendants. Elles prennent alors la forme d'une rétrocession. C'est le cas des fils actifs qui prolongent leur maintien au domicile parental en cas de chômage du père.

Les rapports entre générations peuvent prendre d'autres formes que l'exercice de solidarités familiales. Ils peuvent également mettre en opposition des groupes sociaux pour l'occupation des places sociales et constituent à ce titre des rapports de concurrence. Pour P. Bourdieu (1985), les oppositions entre les générations confronteraient dans un champ social donné les détenteurs de biens ou de positions sociales aux prétendants à ces mêmes biens ou places. Les conflits de génération seraient en fait des conflits de succession pour la transmission du pouvoir. À ce titre, le paradoxe des politiques de gestion des âges de la vie réside dans le fait que même dans leurs mesures les plus catégorielles, elles affectent inévitablement l'ensemble des catégories d'âge, et établissent entre elles des relations de concurrence.

Le dispositif de socialisation professionnelle en direction de la jeunesse a incontestablement eu des répercussions sur l'équilibre de la pyramide des âges à l'emploi. Dans un premier temps, la gestion politique du chômage a mis les jeunes en « vacance de travail », en vertu de l'idée selon laquelle il était moins socialement dommageable pour les jeunes que pour les adultes d'être privés d'emploi. La multiplication des statuts intermédiaires ou d'attente, entre l'école et l'emploi, créés en direction du public juvénile, afin de remédier à l'absence d'une réelle occupation professionnelle, et la fixation d'un plancher légal de rémunération (sorte de sous-«SMIG jeunes») ont ainsi légitimé l'idée que les jeunes pouvaient être mis à l'écart de la vie active proprement dite, et qu'ils avaient des besoins moins importants que les adultes en matière de ressources.

La politique de traitement social de la crise économique a par conséquent semblé, dans un premier temps, se réaliser au détriment de la main-d'œuvre juvénile. La question du chômage des jeunes maîtrisée, celle de leur insertion professionnelle est restée en suspens. Dans un deuxième temps, cependant, les politiques d'aide à l'insertion 
professionnelle des jeunes, avec leur cortège de mesures fiscales et sociales fortement incitatives à l'embauche des jeunes classes d'âge, ont quelque peu fragilisé la situation des chômeurs et des actifs plus âgés, sans pour autant stabiliser celle des plus jeunes. Les mesures adoptées en faveur de l'emploi des jeunes ont eu pour effet d'accélérer les licenciements dans les autres classes d'âge. L'adoption de politiques catégorielles a donc conduit à écarter du marché du travail des catégories de travailleurs plus âgés. Les hommes et les femmes de plus de 50 ans en situation de demandeurs d'emploi n'ont pas bénéficié des effets de la reprise économique alors que la situation des jeunes s'est améliorée à partir de 1985. Le dispositif institutionnel de socialisation des jeunes et, plus globalement, la gestion politique des âges produit ainsi un état de concurrence intergénérationnelle pour l'accès à l'emploi.

«Lorsqu'on définit les phases de la vie par des éléments institutionnels, on peut retrouver dans les institutions mises en place un rôle de médiation dans les rapports entre générations [...] L'institutionnalisation du cours de la vie revient alors à la gestion institutionnalisée des rapports entre générations. " (AttiasDonfut, 1988.)

97 La gestion actuelle des âges de la vie possède donc un aspect contradictoire en assurant à la fois la médiation et l'aménagement des rapports entre générations et en organisant leur concurrence sur le marché de l'emploi.

Une forme plus visible et plus directe des rapports entre générations est celle des conflits qui opposent parents et enfants. Ces conflits ont le plus souvent été expliqués par l'existence d'une décalage culturel entre ces deux générations. Il y aurait, dans ce cas, un fossé des générations (Mead, 1979), qui interdirait l'établissement de tout rapport d'entente ou de solidarité. On peut tout autrement se référer à la notion de crise de succession économique entre les générations pour éclairer ces situations épisodiques de conflit. Les parents oublieraient qu'ils appartiennent à une génération qui a connu des conditions économiques d'insertion socioprofessionnelle différentes de leurs enfants. Les relations intra-familiales constituent ici un domaine privilégié où se déploie la dimension cognitive de l'effet de génération. La conscience de génération s'exercerait ainsi en sens contraire, ou de façon négative, pour prendre la forme d'une " amnésie générationnelle » (Blöss, 1991). Quand la situation sociale de son enfant s'écarte trop sensiblement de celle dans laquelle on était à son âge, la tension familiale est si forte qu'elle peut produire des ruptures dans le processus cognitif des parents, une amnésie dans leur conscience de génération.

99 Le conflit socio-générationnel n'est pas un conflit permanent. Solidarité, conflits, concurrence constituent ainsi autant de modalités des rapports intergénérationnels que la transformation des modes de socialisation redéfinit et recompose. C'est dans ces mutations sociales que la jeunesse prend forme.

\section{Conclusion}

Dans la vision catégorielle des âges de la vie, tant naturaliste (biologique, psychologique) qu'institutionnelle, où la jeunesse est nettement différenciée de l'état adulte en prévision duquel elle est censée se socialiser, toute idée de contagion des questions juvéniles au monde de leurs aînés est d'emblée écartée. Dans la vision sociologique des âges de la vie que nous avons développée dans ce texte, moins fonctionnaliste, l'opposition catégorielle 
entre le monde la jeunesse et celui des adultes s'efface, et inscrit la réflexion sur la jeunesse dans une problématique du changement social.

Pour autant, la jeunesse comme période de l'existence doit-elle être strictement caractérisée par sa dimension conjoncturelle (elle serait une conjoncture biographique au même titre que d'autres étapes du cycle de vie), intermédiaire ou encore transitoire, ou bien les changements que nous observons dans les comportements ou plus largement dans les modes de vie juvéniles, ne traduisent-ils pas des mutations sociales en cours à l'échelle du cycle de vie tout entier? Quand le transitoire (précaire ou non) perdure, qu'en est-il des conséquences sur la structuration même du cours de l'existence? Cette interrogation traverse notre réflexion tout au long de ce texte.

Dans cette vision des âges de la vie, le temps social de la jeunesse n'est pas un temps en soi, ni même un temps directement préparatoire au temps social de l'adultéité. Il constitue, selon un point de vue «barycentrique» un temps qui reçoit son sens sociologique de l'ensemble des temps sociaux du cycle de vie (enfance, âge adulte, vieillesse), et de leurs transformations sociales. Il constitue également un temps actif dont les effets, tant dans les rapports de sexe, d'éducation, que d'emploi, s'apprécieront sur l'ensemble du cycle de vie.

\section{BIBLIOGRAPHIE}

AtTiAs-Donfut C. - Sociologie des générations. L'empreinte du temps, Paris, Presses universitaires de France, 1988. - « Rapports de générations et parcours de vie ", in Biographie et cycle de vie, $\mathrm{n}^{\circ} \mathrm{sp}$. de : Enquête. Cahiers du CERCOM, 5, 1989.

BLöss T. - « Jeunes Maghrébins des quartiers nord de Marseille. Une génération charnière », in Familles, générations, patrimoines, $\mathrm{n}^{\circ} \mathrm{sp}$. de : Les Annales de la Recherche urbaine, 41, 1989, p. 59-66. - « Jeunes, précarité et rapports entre générations. Réflexion à partir de données d'enquête ", in Le fait générationnel, $\mathrm{n}^{\circ} \mathrm{sp}$. de : Annales de Vaucresson, 1991.

BLÖSS T. \& FERONI I. - La mise en forme de la jeunesse. Essai de synthèse critique, Rapport de recherche UfJT, $114 \mathrm{p}$.

BlÖSS T., FRICKEY A. \& GODARD F. - « Cohabiter, décohabiter, recohabiter. Itinéraire de deux générations de femmes ", Revue française de Sociologie, 31, 1990, p. 553-572.

Bouffartigue P., Lagrée J.-C. \& Rose J. - « Jeunes : de l'emploi aux modes de vie. Points de vue sur un champ de recherche », Formation Emploi, 26, 1989.

BOURDIEU P. - « De quoi parle-t-on quand on parle de la jeunesse? », in Les jeunes et les autres, CRIV, 1985.

CENTRE D'ÉTUDE DES REVENUS ET DES COÛTS (CERC) - « Des jeunes en difficulté entre l'école et l'emploi. Le clivage des générations ", in Les Français et leurs revenus. Le tournant des années 80, Paris, La Documentation française, 1989. 
CHAMBOREDON J.-C. - « Adolescence et post-adolescence : la juvénisation. Remarques sur les transformations récentes des limites et de la définition sociale de la jeunesse ", in A.-M. Alléon, O. Morvan, S. Lebovici, eds, Adolescence terminée, adolescence interminable, Colloque national sur la post-adolescence (Grenoble, 30 avril-1 ${ }^{\mathrm{er}}$ mai 1983) Paris, Presses universitaires de France, 1985, p. 13-28.

Commaille J. - « Essai de sociologie politique de la « jeunesse ». Le droit comme miroir », in Les jeunes et les autres, CRIV, 1985, t. 2.

Galland O. - « Précarité et entrées dans la vie », Revue française de Sociologie, 25, 1984, p. 49-66.

GERM-CERCOM - Itinéraires féminins. Les calendriers familiaux, résidentiels et professionnels de deux générations de jeunes femmes dans les Alpes-Maritimes, CNRS (PIRTTEM)/Ministère de la Recherche et de la Technologie (Programme TET), 1989.

GODARD F. - « Cultures et modes de vie de génération en génération », in Les jeunes et les autres, CRIV, 1989, t. 2 - « Sur quelques voies ouvertes par une sociologie des générations », Les Annales de Vaucresson, CRIV, 1991.

Guillemard A.-M. - La vieillesse, une mort sociale, Paris-La Haye, Mouton, 1972.

LAGRÉE J.-C. \& LEW-FAI P. - La jeunesse en questions. Orientations documentaires et sources de recherche en sciences sociales en 1982, Paris, La Documentation française, 1983. - Jeunes et chômeurs, Paris, Presses du CNRS, 1989, 226 p.

MANNHEIM K. - « The problem of generation », in Essays of the sociology of knowledge, Londres, Routledge \& Kegan Paul, 1953.

MARUANi M. \& Nicole C. - Au labeur des dames. Métiers masculins, métiers féminins, Paris, Syros, 1989.

MAUGER G. - «Éléments pour une réflexion critique sur la catégorie de jeunesse », Cahiers Jeunesses et Sociétés, 6-7-8, 1985a. - "Le phénomène des blousons noirs ", in Colloque de la Société d'Ethnologie française, Le Creusot, 1985b. - « Les héritages. Éléments pour une analyse des rapports entre " générations familiales » ", in S. Magri \& M. Freyssinet, eds, Les rapports et leurs enjeux, Paris, CNRS, Cultures et sociétés urbaines (Csu), 1989.

MEAD M. - Le fossé des générations, Paris, Denoël, 1979.

PASSERON J.-C. - « L'inflation des diplômes », Revue française de Sociologie, 23 (4), 1982.

Prost A. - L'enseignement s'est-il démocratisé ?, Paris, PUF, 1986.

SCHWARTZ, L. - L'insertion professionnelle et sociale des jeunes. Rapport au Premier ministre, Paris, Documentation française, 1981.

THÉLот C. - « Le sous-emploi a doublé en quatre ans », Économie et Statistique, 193-194, 1986.

THÉVEnot L. - « Une jeunesse difficile. Les fonctions sociales du flou et de la rigueur dans les classements ", Actes de la Recherche en Sciences sociales, 26-27, 1979. - « Grandeurs et misère de la jeunesse : la qualité du jeune dans les jugements de grandeur ", in Les jeunes et les autres, CRIV, 1985.

WIDMER J. - « Remarques sur les classements d'âge », Revue suisse de Sociologie, 9-2, 1983. 


\section{NOTES}

1. «Une observation des seuils d'âge légaux pour ce qui concerne le statut personnel du "jeune", son statut dans ou par rapport à sa famille, sa vie sexuelle, sa possibilité de constituer une famille par le mariage, son insertion et son statut par le travail, son engagement dans l'armée, sa responsabilité civile, ses possibilités de conduire un véhicule, ses possibilités d'accomplir des actes de la vie quotidienne, son statut dans les loisirs (pêche, chasse, sports, cinéma, cafés), révèle que l'unicité de l'âge donnant accès à la majorité civile s'accompagne en fait d'une pluralité d'âges donnant accès à des droits ou des possibilités au "jeune" (de 13 à 18 ans tous les âges sont ici sollicités). Ces seuils sont institués par un grand nombre de droits ou d'institutions : du Code civil ou du Code du travail au Code des débits et boissons ou au Code rural ou au Code du service national, etc. »

2. Entre 1975 et 1982 les politiques de l'emploi en direction des jeunes prennent la forme de dispositifs transitoires ou de plans conjoncturels de formation. Seront mises en place successivement plusieurs formules: Stages Granet; Pactes pour l'Emploi ; Contrats Formation Insertion et Formation Qualification.

La période 1982-1986 est marquée par la mise en œuvre de la politique d'aide à l'insertion sociale des jeunes à la suite de la parution du Rapport Schwartz. Les structures essentielles en seront les PAIO et les Missions locales. La mise en place des TUC et des SIVP complétera le dispositif.

3. Cf. la mise en place successive des procédures " Habitat et vie sociale " et " Développement social des quartiers ", en quartiers populaires. La mise en place des ZEP illustre par ailleurs la volonté d'articulation des instances de socialisation scolaire au niveau local.

4. Interruption du bénéfice des « allocations logement » pour un jeune travailleur après 25 ans ; limite d'âge atteinte, toujours à 25 ans, pour prétendre à un logement en FJT, etc.

5. Entre 1983 et 1988 la population scolarisée âgée de 16 et 25 ans s'est globalement accrue de 500000 jeunes. Les effectifs scolaires et universitaires atteignent 3,3 millions en 1988 contre 2,8 en 1983 (Source: Données sociales, 1990). Ce qui se traduit par une élévation du taux de taux scolarisation : de 32,5\% en 1983 pour les $16-25$ ans, il passe à $39 \%$, en 1988, pour cette même classe d'âge. Cette augmentation sensible de la population scolarisée est fortement liée à la progression des effectifs lycéens (qu'il s'agisse de la filière générale ou professionnelle) et universitaires.

6. De plus en plus d'élèves poursuivent leurs études au-delà de 16 ans : les sorties à 17,18 et 19 ans se sont accrues au dépend des plus jeunes. $90 \%$ des jeunes sont scolarisés entre 16 et 17 ans et $40 \%$ entre 18 et 21 ans. C'est d'ailleurs pour cette dernière classe d'âge que la progression de la scolarisation a été la plus importante.

7. 442329 contre $401575 \mathrm{chez}$ les garçons (Source : Données sociales, 1990).

8. Le niveau $\mathrm{V}$, qui comprend les sorties de l'année terminale des cycles courts et les abandons en cours de second cycle long, voit sa part demeurer à peu près inchangée entre 1973 et 1988, et continue à regrouper la plus grosse proportion des effectifs sortants, soit $44 \%$ pour la dernière année de référence. Les sorties aux niveaux extrêmes ont au contraire sensiblement évolué au cours de la même période. Les sorties au niveau le plus bas, niveau VI (sorties du premier cycle du second degré - sixième, cinquième, quatrième - et des formations pré-professionnelles en un an - CEP, CPPN et CPA -), sont en baisse régulière : 8,4 \% en 1973 contre 4,6 \% en 1986. Les sorties de l'enseignement supérieur ont, quant à elles, rapidement augmenté pour passer de $8,4 \%$ en 1973 à 33,5 \% en 1986.

9. Neuf mois après la sortie de l'école, les situations sont en effet très différenciées selon le niveau de diplôme : 30 \% des titulaires d'un CAP ou d'un BEP doivent se contenter d'un TUC ou 
d'un SIVP ( $14 \%$ pour les garçons et $42 \%$ pour les filles); seulement $3 \%$ des diplômés d'enseignement en sont réduits à cette " extrémité" de forme d'emploi.

10. Le taux d'activité professionnelle des $16-25$ ans est en effet passé de $55 \%$ en 1983 à $49,9 \%$ en 1988.

11. Au début des années 1980 , leur taux de chômage est très élevé, et nettement supérieur à celui des adultes. En 1981 les 16-21 ans, qui représentent $8 \%$ de la population active, composent $28 \%$ des demandeurs d'emploi. En 1982, 39 \% des chômeurs ont moins de 25 ans. Fin 1985, 20 \% des jeunes actifs et $28 \%$ des jeunes actives sont au chômage, contre $6 \%$ des hommes adultes et $10 \%$ pour les femmes adultes.

12. Un jeune sur six occupe en 1988 un emploi « aidé ».

13. Alors que la part des emplois précaires (intérim, contrat à durée déterminée, stages) est faible parmi l'ensemble de la population active, tous âges confondus : $4,8 \%$ des salariés sont dans ce cas en 1986, la proportion atteint $16,5 \%$ parmi les moins de 25 ans. Ces données sociales pèsent d'un poids d'autant plus lourd qu'il existe une forte proximité entre ces types d'emploi et la probabilité de «tomber au chômage ». Chez les hommes comme chez les femmes la précarité de l'emploi est la seconde cause de chômage, juste après le licenciement: un jeune chômeur sur trois provient d'un emploi précaire.

14. "Est en situation de sous-emploi, toute personne travaillant moins que la norme ou que d'habitude et cherchant un autre travail à temps complet ». Définition donnée par C. Thélot (1986).

15. À l'échelle nationale ou lors d'enquêtes sur des bassins d'emploi localisés.

16. Si on a effectivement constaté que les jeunes chômeurs résident plus longtemps dans leurs familles que les jeunes actifs, on a pu également noter que l'accroissement de la proportion des chômeurs de 18 à 24 ans habitant chez leurs parents n'est pas à la mesure de l'accroissement du nombre des chômeurs dans cette même classe d'âge.

17. Nous avons pu constater, dans une enquête récente (1989), que les conditions de départ du domicile familial constituaient pour les femmes une "variable explicative » de leur futur profil d'activité socioprofessionnelle. Quitter ses parents pour se marier augure un cycle de vie principalement centré sur la famille, où l'activité féminine ne s'inscrit que dans le tissu intersticiel des charges domestiques et de la carrière professionnelle du conjoint. En revanche, quitter ses parents pour prendre un emploi ou pour suivre une formation conduit les femmes vers une présence plus continue sur le marché de l'emploi, et corrélativement à des positions sociales plus élevées. Cf. T. Blöss \& A. Frickey, «L'activité féminine ou l'impossible "double carrière" ", Notes et Documents du CERCOM, mai 1991, à paraître).

18. La sociologie des générations peut être perçue de nos jours comme un chantier théorique récemment réactivé. Dans ces conditions, notre propos aura essentiellement pour vocation d'indiquer les principales orientations de recherches qui conjuguent les concepts de jeunesse et de génération, plutôt que d'établir un bilan définitif des contributions de l'approche générationnelle à la catégorie de jeunesse.

19. Comparaison intergénérationnelle de femmes nées respectivement en 1947 et 1959, dans le cadre dune enquête menée au GERM-CERCOM, et ce, à partir d'hypothèses de ruptures sociohistoriques dans les comportements de passage à l'âge adulte (1989).

20. Méthodes d'analyse rappelées par C. Attias-Donfut (1988).

21. Cf. notamment Mannheim (1953).

22. Dans une enquête ayant pour objet les jeunes des quartiers nord de Marseille : cf. T. Blöss, J'ai hérité de la misère, MELATT/Conseil régional PACA, 1987. 